\title{
The prospects of reflectometry and ellipsometry with Colorado State University tabletop XUV laser
}

\author{
I.A. Artioukov, B.R. Benware ${ }^{1}$, R.M. Fechtchenko, J.J. Rocca ${ }^{1}$, M. Seminario', \\ A.V. Vinogradov and M. Yamamoto ${ }^{2}$ \\ P. N. Lebedev Physical Institute, 53 Leninsky Prospekt, Moscow 117924, Russia \\ ${ }^{1}$ Colorado State University, Electrical Engineering Department, Fort Collins, CO 80523, U.S.A \\ ${ }^{2}$ Research Institute for Scientific Measurements, Tohoku University, 2-1-1 Katahira, Aoba-ku, \\ Sendai 980 , Japan
}

\begin{abstract}
The capillary discharge ArIX laser (wavelength $46.9 \mathrm{~nm}$ ) is a new device attractive for various applications due to its unique for XUV lasers properties: high average power and compactness. Reflectometry and ellipsometry are among them. The first is capable to provide fundamental data on optical constants of solids, which are still rather limited. Reflection coefficient in this spectral range is highly sensitive to the presence of an overlayer at the surface of a sample. This hinders bulk optical constants determination, but offers the possibility to study chemical contamination by ambience. The theory of reflection from a surface with a thin overlayer is developed. It enables to rigorously include overlayer into reflectometry data processing and derive separately the parameters of bulk and overlayer. Ellipsometry in general is more powerful than reflectometry method to measure optical constants. In addition it offers the accurate methods to measure film thickness and properties of magnetic materials. Unfortunately high quality analyzers and polarizers are not available in this spectral range, which requires specific approach to ellipsometric measurements. We discuss and use for this purpose $\mathrm{Sc} / \mathrm{Si}$ multilayer structures.
\end{abstract}

\section{INTRODUCTION}

The compact capillary discharge laser at $46.9 \mathrm{~nm}$ developed in Colorado State University [1] has reached peak power $0.6 \mathrm{MW}$ and average power $3.5 \mathrm{~mW}$ [2]. The main laser parameters are shown in Table 1. They are sufficient for various experiments and measurements, some of which were reported in [3]$[6]$.

Table 1. The parameters of tabletop Ne-like Ar capillary discharge soft $x$-ray laser.

\begin{tabular}{|c|c|}
\hline Parameter & Value \\
\hline Wavelength & $46.9 \mathrm{~nm}$ \\
\hline Pulse energy & $0.1-1.0 \mathrm{~mJ}$ \\
\hline Pulse duration & $0.6-1.5 \mathrm{~ns}$ \\
\hline Average power & $3.5 \mathrm{~mW}$ \\
\hline Peak power & $0.1-0.6 \mathrm{MW}$ \\
\hline Bandwidth $\Delta \lambda \lambda$ & $<10^{-4}$ \\
\hline Spatial Coherence & $4 \mathrm{~mm}$ at $6 \mathrm{~m}$ \\
\hline Size & $6 \times-8 \times$ Diffraction Limited \\
\hline
\end{tabular}

Multilayer $\mathrm{Sc} / \mathrm{Si}$ coatings developed in Moscow X-ray Optics Group [7,8] allow increasing considerably (up to $45 \%$ ) reflectivity in this wavelength range (see fig. 1), which is also helpful for applications of $46.9 \mathrm{~nm}$ laser. 


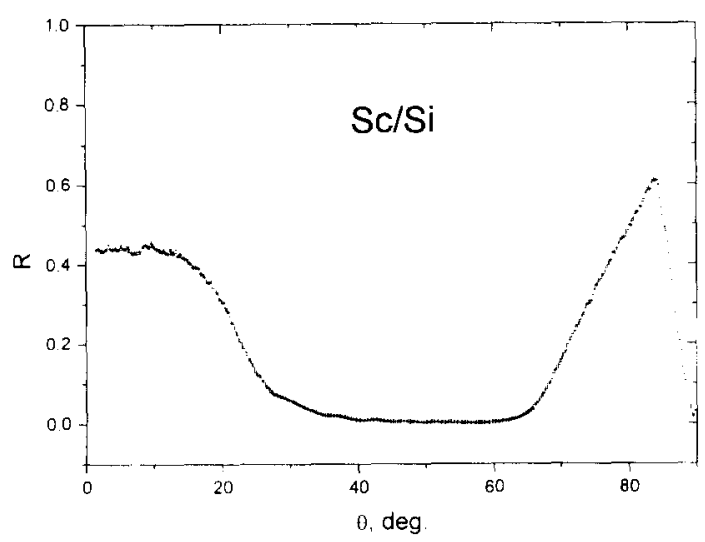

Figure 1. Reflectivity of a $\mathrm{Sc} / \mathrm{Si}$ multilayer mirror as function of incidence angle.
In this paper we discus the prospects of applications of CSU laser in reflectometry and ellipsometry.

\section{REFLECTOMETRY}

An important parameter responsible for interaction of radiation with matter is a penetration depth. For various of lasers actions such as surface processing and modification, annealing, ablation, plasma formation and dynamics the normal incidence of laser beam is employed. In this case the penetration depth equals to:

$$
l_{n}=\frac{\lambda}{4 \pi \kappa}
$$

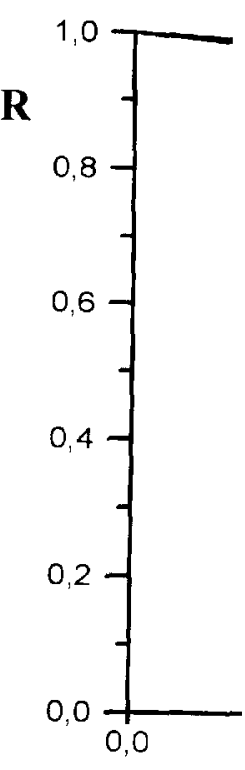

where $\varepsilon=1-\delta+i \beta=(n+i \kappa)^{2}-$ complex dielectric function of a sample.

On the other hand, if we are interested in the structure of near surface layers, contamination by ambiance gases etc. the oblique incidence as in X-ray reflectometry $[9,10]$ can be used. In this case the important value is the penetration depth for incidence at the critical angle $\theta_{c}=\pi / 2-\arcsin \sqrt{\delta}$ :

$$
l_{c a}=\frac{\lambda}{4 \pi \sqrt{n \kappa}}
$$

Table 2 shows $l_{n i}$ (see (1)) and $l_{c u}$ (see (2)) at laser wavelength for various materials in comparison with the same values at $0.154 \mathrm{~nm}$ regularly used in $\mathrm{X}$-ray reflectometry.

Table 2. The penetration depths for different materials at the wavelengths $46.9 \mathrm{~nm}$ and $0.154 \mathrm{~nm}$.

\begin{tabular}{|c|c|c|c|c|c|c|c|c|c|}
\hline & & $C$ & $\mathrm{Si}_{3} \mathrm{~N}_{4}$ & $\mathrm{SiO}_{2}$ & $\mathrm{SiC}$ & $\mathrm{Si}$ & $\mathrm{InP}$ & $\mathrm{GaAs}$ & Ir \\
\hline \multirow{2}{*}{$l_{m i}$} & $\lambda=46.9 \mathrm{~nm}$ & $5.1 \mathrm{~nm}$ & $9.3 \mathrm{~nm}$ & $11.7 \mathrm{~nm}$ & $16.0 \mathrm{~nm}$ & $129 \mathrm{~nm}$ & $41.5 \mathrm{~nm}$ & $62.0 \mathrm{~nm}$ & $7.0 \mathrm{~nm}$ \\
\cline { 2 - 23 } & $\lambda=0.154 \mathrm{~nm}$ & $766 \mu \mathrm{m}$ & $74 \mu \mathrm{m}$ & $130 \mu \mathrm{m}$ & $70 \mu \mathrm{m}$ & $80 \mu \mathrm{m}$ & $11 \mu \mathrm{m}$ & $28 \mu \mathrm{m}$ & $2 \mu \mathrm{m}$ \\
\hline \multirow{2}{*}{$l_{c a}$} & $\lambda=46.9 \mathrm{~nm}$ & $6.0 \mathrm{~nm}$ & $7.1 \mathrm{~nm}$ & $8.3 \mathrm{~nm}$ & $11.0 \mathrm{~nm}$ & $27.0 \mathrm{~nm}$ & $13.0 \mathrm{~nm}$ & $17.0 \mathrm{~nm}$ & $5.7 \mathrm{~nm}$ \\
\cline { 2 - 11 } & $\lambda=0.154 \mathrm{~nm}$ & $97.0 \mathrm{~nm}$ & $30.2 \mathrm{~nm}$ & $40 \mathrm{~nm}$ & $30.0 \mathrm{~nm}$ & $32.0 \mathrm{~nm}$ & $11.5 \mathrm{~nm}$ & $5.9 \mathrm{~nm}$ & $5.4 \mathrm{~nm}$ \\
\hline
\end{tabular}

As is seen from Table 2 the penetration depths for $49.6 \mathrm{~nm}$ are very short especially for light elements $\mathrm{C}, \mathrm{O}, \mathrm{N}$ and heavy metals - shorter than in any other spectral range including $0.154 \mathrm{~nm}$. Therefore reflectivity is expected to be very sensitive to the presence of ambient gases and compounds, and radiation of CSU laser can be used to reveal and control the presence and formation of carbides, oxides and nitrides on a surface. For example $1 \mathrm{~nm}$ thick $\mathrm{SiO}_{2}$ film gives considerable correction to reflectivity of clean $S i$. The reflectivities of clean and oxidized silicon are compared in fig.2 in XUV (46.9 nm) and hard $X$-rays $(0.154 \mathrm{~nm})$. Due to this highest sensitivity, the surface contamination hinders determination of optical constants of solids in the XUV.
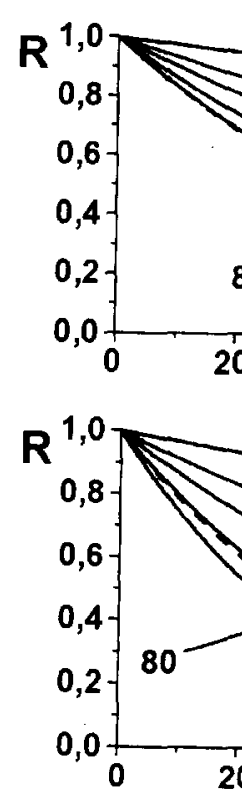

Figure2. Ref indicate film 
prospects reflecto-

sible for atter is a of lasers sing and

1, plasma mal incid. In this to:

(1) by ambire impor-

(2)

mparison

\begin{tabular}{|c|}
\hline $\mathrm{Ir}$ \\
\hline $7.0 \mathrm{~nm}$ \\
\hline $2 \mu \mathrm{m}$ \\
\hline $5.7 \mathrm{~nm}$ \\
\hline $5.4 \mathrm{~nm}$ \\
\hline
\end{tabular}

light elen. There$\mathbf{s}$, and raxides and sctivity of ( nm) and rmination

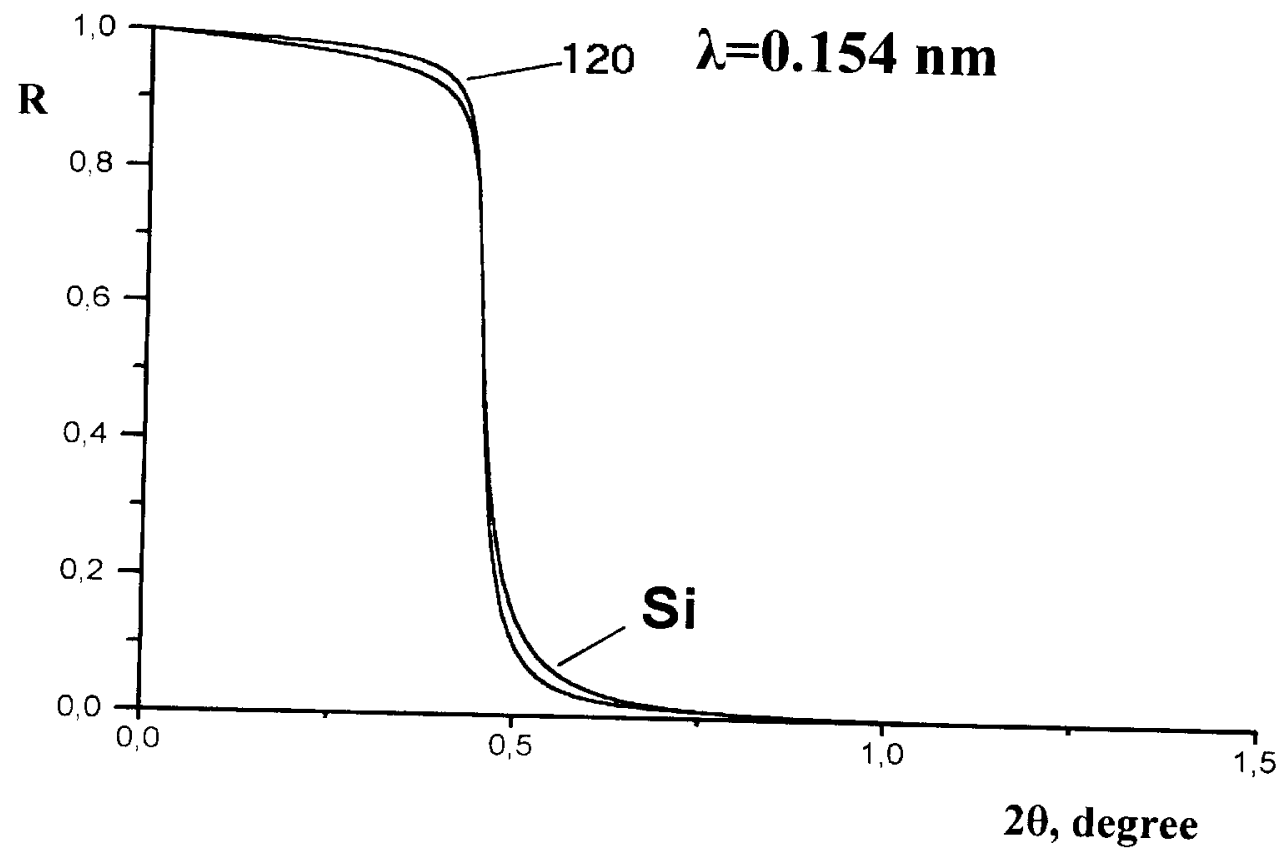

$\lambda=46.9 \mathrm{~nm}$
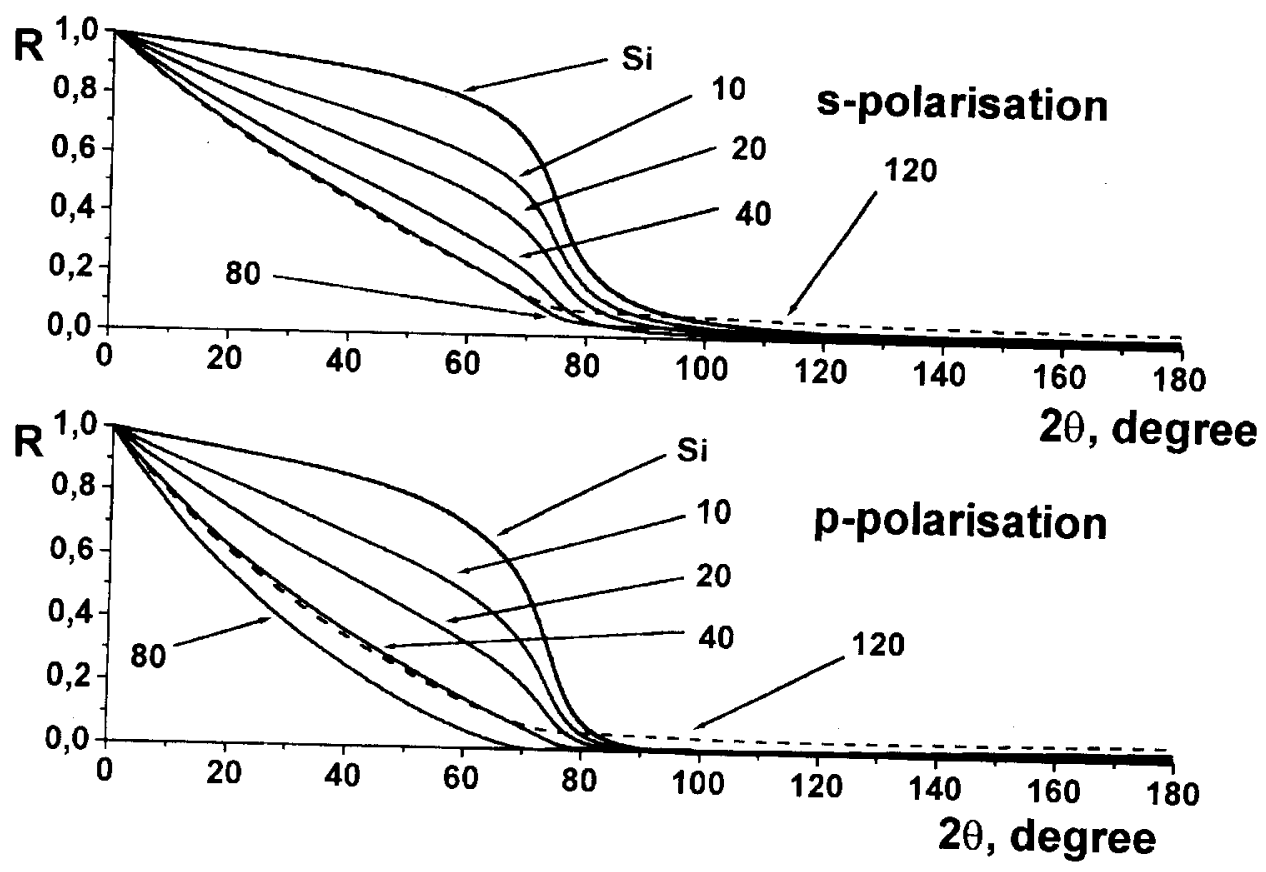

Figure2. Reflectivity of Si covered with oxide $\mathrm{SiO}_{2}$ film at $0.154 \mathrm{~nm}$ and $46.9 \mathrm{~nm}$. Numbers $(10,20 \ldots)$ indicate film thickness in $\AA$. 


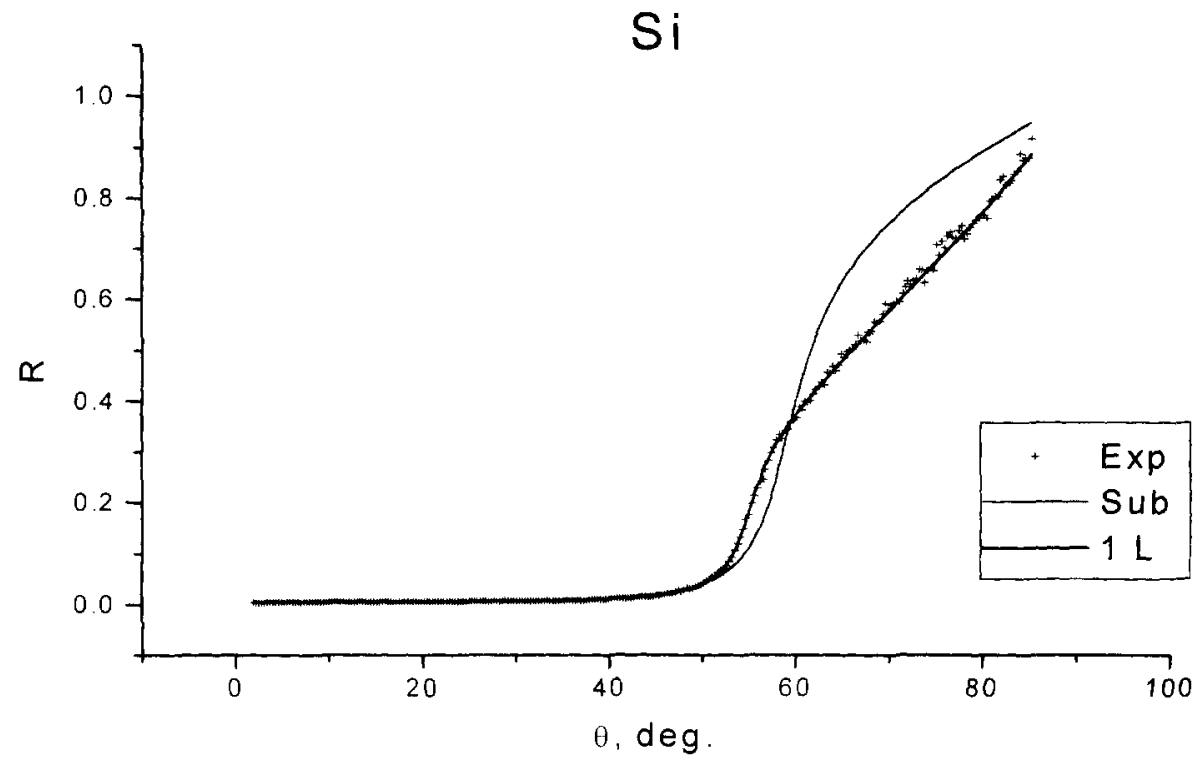

Figure 3. Reflectivity data for $S i$ at $46.9 \mathrm{~nm}$ and results of fitting procedure Thin solid line - Fresnel formula for a clean surface, thick solid line - Fresnel formula with the account for contamination layer.

Figure 3 gives an example of reflectivity data obtained with CSU laser. To reach the agreement between the measured reflectivity and Fresnel formulas we had to assume the presence of transition layer on the silicon surface. The fitting of theoretical and measured reflectivity curves enables to find optical constants $\delta$ and $\beta$ of pure $S i$ with the account for contamination layer [3]. Similar measurements have been made with CSU laser for GaAs, GaP, InP, GaAsP and Ir [3]. More accurate approaches to the problem based on the reflection theory are discussed in [11]-[13].

\section{ELLIPSOMETRY}

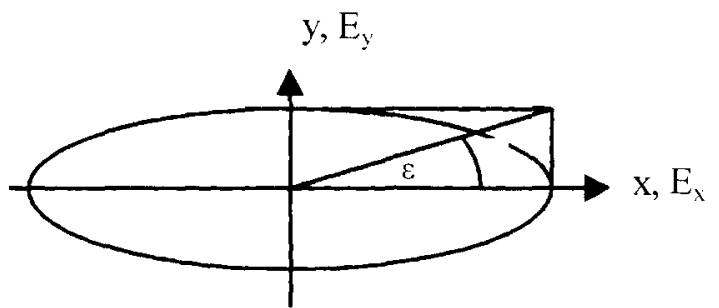

Figure 4. The scheme showing the main parameters of a polarized beam.
Ellipsometry implies the full control of beam parameters: intensity $\mathrm{I}$; degree of polarization $\mathrm{P}$; elleptisity angle $\varepsilon$ (see fig. 4); and the angle $\theta^{\prime}$, characterizing the spatial orientation of polarization ellipse. Ellipsometry enables: (a) to measure mentioned parameters before and after reflection from a sample; (b) to produce linearly or circularly polarized beams; (c) to determine phase difference between reflection coefficients for $s$ and p polarized waves. Ellipsometry is an alternative method (often more accurate than reflectometry) to measure optical constants of solids. Ellipsometry is also a unique method to investigate the structure of magnetic and anisotropic materials and to measure thin film thicknesses. Evidently ellipsometrical methods can considerably enlarge the field of X-

\footnotetext{
${ }^{1}$ Note that $\varepsilon$ and $\theta$ denote in this section other physical values than in section 2.
}

ray laser applications.

Multilayer structures at $45 \mathrm{~d}$ to provide half and quarter wave soft $\mathrm{X}$-ray range have been made tory laser-produced plasma sourc

To start polarization experin flectivity at $45^{\circ}$ of incidence we shown on fig.6. The goal was to ings calibration. Two multilayer

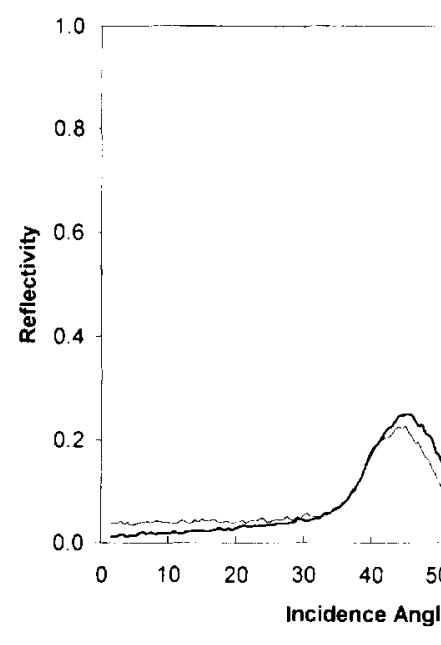

Figure 5. The reflectivities of two $\mathrm{m}$ ments.

by proper design of multilayers only one multilayer used as a pol some errors, which are still diffic are available and usually are far fr cess with the use the of full Stoke tions between the parameters of in

$$
I^{\prime}=
$$

$\cos 2 \theta^{\prime}$

where the angles $\theta$ and $\theta^{\prime}$ denote sition of the reflector; $Y$ is the inte

These formulas can be used 
ray laser applications.

Multilayer structures at 45 degrees of incidence in reflection or transmittion modes have been shown to provide half and quarter wave plates for soft X-rays [14]. So far most of ellipsometry measurements in soft X-ray range have been made at synchrotron radiation facilities and only a few [15]-[17] with laboratory laser-produced plasma sources.

To start polarization experiments with CSU laser [18] two $\mathrm{Sc} / \mathrm{Si}$ multilayer mirrors optimized in reflectivity at $45^{\circ}$ of incidence were fabricated (see fig. 5). The scheme of the polarization experiment is shown on fig.6. The goal was to produce a linearly polarized beam and then to use it for diffraction gratings calibration. Two multilayer mirrors are shown in fig. 6 as polarizer and analyzer. The laser was con-

1.0

0.8

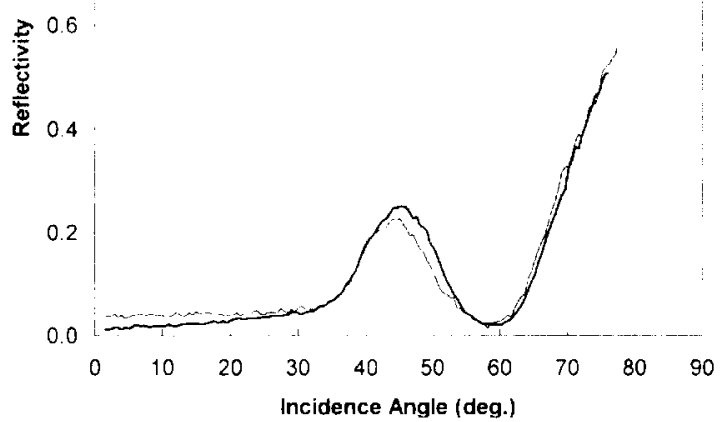

Figure 5. The reflectivities of two mirror used in the experiments. tinuously fired at a repetition rate $1 \mathrm{~Hz}$ while analyzer mirror was rotated from 0 to 180 degrees.

The resulting signal at the detector is shown in fig.7. Analysis of this signal together with reflectivity measurements of $\mid R^{2}=\left(\left|R_{s}\right|^{2}+\left|R_{p}\right|^{2}\right) / 2$ allowed predicting degree of polarization of the outgoing beam, which is $96 \%$ (when the mirrors are parallel) even if the primary laser beam is completely unpolarized. In spite of two mirrors involved, the high average laser power provided enough intensity to produce grating calibration for linearly polarized beam [18].

This was achieved with rather small values of $\left.\rho\right|^{2}=\left|R_{s}\right|^{2} /\left.R_{p}\right|^{2}=7$. Calculations show that much higher values of $|\rho|^{2}$ can be obtained

by proper design of multilayers [19]. In this case for the same type of measurements one can get with only one multilayer used as a polarizer. However in practice the optical constants of Sc are known with some errors, which are still difficult to estimate and one have to work with the synthesized mirrors that are available and usually are far from ideal quarter and half wave plates. The polarization data can be process with the use the of full Stokes parameter formalism [20]. A useful alternative is a set of explicit relations between the parameters of incident and outgoing waves for an arbitrary reflector:

$$
\begin{gathered}
I^{\prime}=I \frac{\left|R_{s}\right|^{2}+\left|R_{p}\right|^{2}}{2} Y, \quad Y=1+P \frac{|\rho|^{2}-1}{|\rho|^{2}+1} \cos 2 \varepsilon \cos 2 \theta \\
P^{\prime}=\sqrt{1-\left[\frac{2 \mid \rho}{Y\left(|\rho|^{2}+1\right)}\right]^{2}\left(1-P^{2}\right)} \\
\sin 2 \varepsilon^{\prime}=\frac{2 P}{Y\left(|\rho|^{2}+1\right) P^{\prime}}(\operatorname{Re} \rho \sin 2 \varepsilon+\operatorname{Im} \rho \cos 2 \varepsilon \sin 2 \theta), \\
\cos 2 \theta^{\prime}=\frac{1}{P^{\prime} \cos 2 \varepsilon^{\prime}}\left[\frac{2|\rho|^{2}}{Y\left(|\rho|^{2}+1\right)}(P \cos 2 \varepsilon \cos 2 \theta+1)-1\right]
\end{gathered}
$$

where the angles $\theta$ and $\theta^{\prime}$ denote orientation of ellipse of incident and reflected beams relative to the position of the reflector; $Y$ is the intensity contrast factor (see fig. 7).

These formulas can be used to analyze the evolution of parameters of a beam travelling through the 
optical system. Another reason for X-ray laser based ellipsometry is the possibility to control very thin films of magnetic materials. This is anticipated due to strong coupling of $46.9 \mathrm{~nm}$ radiation with matter as it was discussed in section 2.

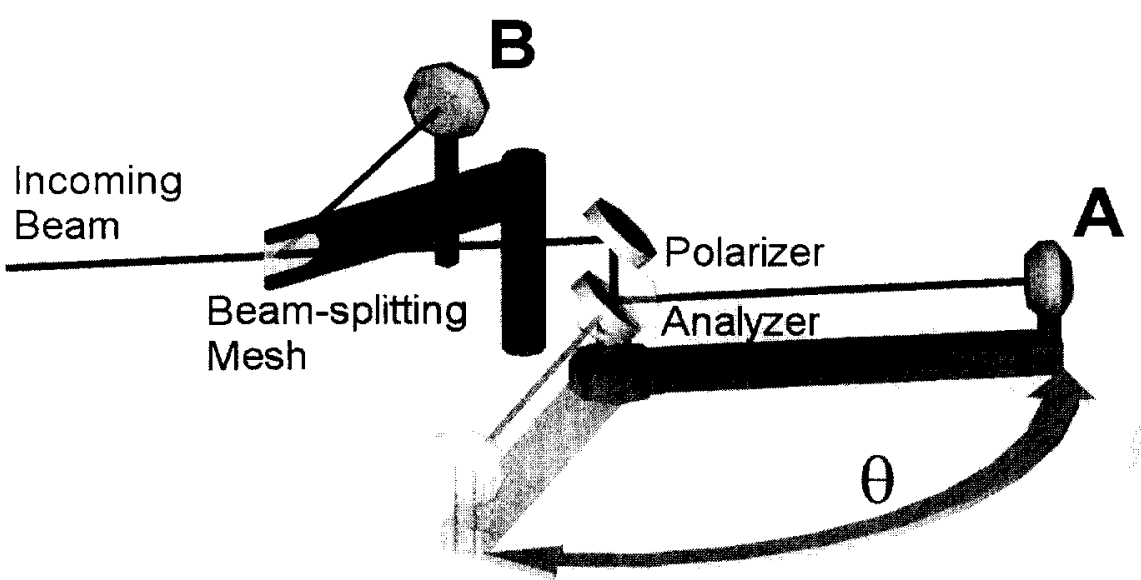

Figure 6. The scheme of the experimental setup.

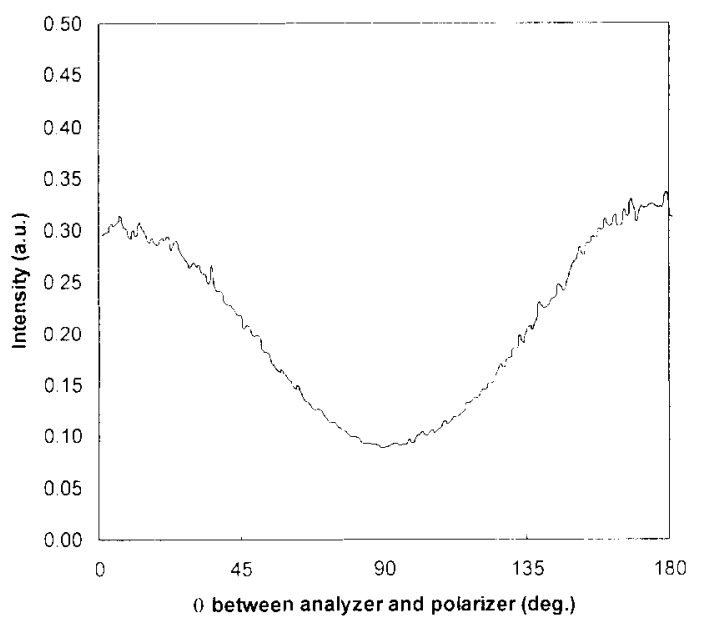
beam outgoing from the optical system as function of rotation angle.
Figure 7. The experimentally measured intensity of the

\section{SUMMARY}

In conclusion the reflectometry and ellipsometry with CSU compact discharge pumped soft X-ray laser are now demonstrated. The expected application fields are metrology and testing of XUV optics, the measurement of optical constants of solids, and investigation of the structure and properties of magnetic materials. Further improvement of the quality of polarizing elements is desirable and looks possible.

\section{Acknowledgment}

The authors are indebted to Dr. Yu.A. Uspenskii for lots of fruitful discussions. This work was supported under ISTC grant No 1051 and by the Physics Educational Science Center "Fundamental Optics and

\section{References}

[1] J.J. Rocca, V.N. Shlyaptse Phys.Rev.Lett., 73 (1994) 219

[2] B.R. Benware, C.D. Macchiet

[3] I.A .Artioukov, B.R. Benwar 1501.

[4] C.H. Moreno, M.C. Marconi, shin, Phys.Rev. E, 60, No 1 (1

[5] C.H. Moreno, M.C. Marconi,

[6] B.R. Benware, A. Ozols, J.J. 24 (1999) 1714-1716.

[7] Yu. A. Uspenski, S.V. Anton transition metals in the spect 3256 (1997) 288-294.

[8] Yu.A. Uspenskii, V.E. Levas shin, E.N. Zubarev, and V.Yu.

[9] A. V. Vinogradov, A.G. Tour 20 No 2 (1999) 136-151.

[10] A. V. Vinogradov, A.G. ? for characterization of supersn

[11] R.M. Fechtchenko, A.V. (2000) 62-68.

[12] R.M. Fechtchenko, A.V. P

[13] R.M.Fechtchenko, A.V.Po

[14] D. Attwood, Soft X-ray an University Press, 1995).

[15] M. Yamamoto, K. Mayan SPIE 1720 (1992) 390-394.

[16] J. B. Kortright, H. Kimur Phys. Lett., 60 (24) (1992) 296

[17] M. Yamamoto, H. Nomur Rel. Phenom., 101-103 (1999)

[18] B.R. Benware, M. Semin Bach, submitted to Journal of [19] R. M. Fechtchenko, A.V. compact discharge X-ray laser

[20] R. M. A. Azzam and N. 1987) 119.

Spectroscopy" in context of Federal Target Program "Integration". 
very thin matter as

netry with $i$ laser are ion fields the measinvestigaretic mateof polariz2.

spenskii for s supported hysics EduOptics and

\section{References}

[1] J.J. Rocca, V.N. Shlyaptsev, F.G. Tomasel, O.D. Cortazar, D. Hartshom, and J.L. Chilla Phys.Rev.Lett., 73 (1994) 2192-2195.

[2] B.R. Benware, C.D. Macchietto, C.H. Moreno and J.J. Rocca, Phys.Rev.Lett., 81 (1998) 5804-5807.

[3] 1.A .Artioukov, B.R. Benware, J.J. Rocca et al, IEEE, J. of Sel. Topics in Q. Elect., 5 (1999) 14951501.

[4] C.H. Moreno, M.C. Marconi, K. Kanizay, J.J. Rocca, Yu.A. Uspenskii, A.V. Vinogradov, Yu.P. Pershin, Phys.Rev. E, 60, No 1 (1999) 911-917.

[5] C.H. Moreno, M.C. Marconi, K. Kanizay, and J.J. Rocca, Opt. Lett., 24 (1999) 420-426.

[6] B.R. Benware, A. Ozols, J.J. Rocca, I.A. Artioukov, V.V. Kondratenko, A.V. Vinogradov, Opt. Lett., 24 (1999) 1714-1716.

[7] Yu. A. Uspenski, S.V. Antonov, V. Yu. Fedotov, and A.V. Vinogradov, "Optical properties of 3dtransition metals in the spectral interval of interest for discharge pumped XUV lasers", Proc. SPIE, 3256 (1997) 288-294.

[8] Yu.A. Uspenskii, V.E. Levashov, A.V. Vinogradov, A.I. Fedorenko, V.V. Kondratenko, Yu.P. Pershin, E.N. Zubarev, and V.Yu. Fedotov, Opt. Lett., 23 (1998) 771-773.

[9] A. V. Vinogradov, A.G. Touryansky, I.V. Pirshin, R.M. Fechtchenko, J. of Russian Laser Research, 20 No 2 (1999) 136-151.

[10] A. V. Vinogradov, A.G. Touryansky, I.V. Pirshin, R.M. Fechtchenko "Two wave X-ray method for characterization of supersmooth substrates", SPIE Proc., 3773 (1999) 167-178.

[11] R.M. Fechtchenko, A.V. Popov and A.V. Vinogradov, J. of Russian Laser Research, 21 No 1 (2000) 62-68.

[12] R.M. Fechtchenko, A.V. Popov and A.V. Vinogradov, , Opt. Lett., 25 (2000) pp.

[13] R.M.Fechtchenko, A.V.Popov and A.V.Vinogradov, J.Phys. B, 2000(submitted).

[14] D. Attwood, Soft X-ray and extreme ultraviolet radiation (Principles and applications, Cambridge, University Press, 1995).

[15] M. Yamamoto, K. Mayama, H. H. Nomura, H. Kimura, M. Yanagihara and T. Namioka, Proc. SPIE 1720 (1992) 390-394.

[16] J. B. Kortright, H. Kimura, V. Nikitin, K. Mayama, M. Yamamoto, and M. Yanagihara, Appl. Phys. Lett., 60 (24) (1992) 2963-2965.

[17] M. Yamamoto, H. Nomura, M. Yanagihara, M. Furudate, and M. Watanabe, J. Electron Spectr. Rel. Phenom., 101-103 (1999) 869-873.

[18] B.R. Benware, M. Seminario, A.L. Lecher, J.J. Rocca, Yu.A. Yspenskii, A.V. Vinogradov, B. Bach, submitted to Journal of Optical Society of America A.

[19] R. M. Fechtchenko, A.V. Vinogradov, "Polarization elements for controlling of a beam of the compact discharge X-ray laser" (see this volume).

[20] R. M. A. Azzam and N. M. Bashara, Ellipsometry and Polarized Light (Elsevier, Amsterdam, 1987) 119 . 